\title{
Counterexamples to a Conjecture on Bounds for the Loss Probability in Finite-Buffer Queues
}

\author{
Yiqiang Q. Zhao \\ Department of Mathematics and Statistics \\ University of Winnipeg \\ Winnipeg, Canada R3B 2E9
}

September 2, 2004

\begin{abstract}
In this paper, we provide counterexamples to a conjecture, made by Miyazawa and Tijms [5], on the upper and lower bounds for the loss probability in finite-buffer queues.
\end{abstract}

Keywords: finite-buffer queues; loss probability; approximations; upper and lower bounds

\section{Introduction}

Consider the finite-buffer $G I / G / c / N+c$ queue, where any arriving customer finding all $c$ servers busy and all $N$ other waiting places occupied is lost. It is assumed that the traffic intensity $\rho=\lambda /(c \mu)<1$, where $1 / \lambda$ is the mean interarrival time and $1 / \mu$ is the mean service time of a customer. The loss probability $P_{\text {loss }}$ is defined as the long-run fraction of customers that are lost.

A number of researchers have proposed approximations to the loss probability using probabilities for the corresponding infinite-buffer queues. Let $\left\{q_{i}\right\}$ and $\left\{q_{i}^{-}\right\}$be the equilibrium probability distributions of the number of customers in the system at an arbitrary point in time and just prior to an arrival epoch, respectively, for the corresponding infinite-buffer queue. Sakasegawa, Miyazawa and Yamazaki [6] proposed the following approximation to 
the loss probability based on $\left\{q_{i}\right\}$ :

$$
P_{\text {appr }}(\text { time })=\frac{(1-\rho)\left(1-\sum_{i=0}^{N+c-1} q_{i}\right)}{1-\rho+\rho \sum_{i=0}^{N+c-1} q_{i}}
$$

and Tijms [7] proposed another approximation based on $\left\{q_{i}^{-}\right\}$:

$$
P_{\text {appr }}(\text { cust })=\frac{(1-\rho)\left(1-\sum_{i=0}^{N+c-1} q_{i}^{-}\right)}{1-\rho+\rho \sum_{i=0}^{N+c-1} q_{i}^{-}} .
$$

These two approximations have been extended to the batch-arrival $G I^{X} / G / c / N+c$ queue by Tijms [7] and Miyazawa and Tijms [5]. The loss probability was also studied by Gouweleeuw [3]. Most of the up-to-date references on this issue can be found in [3]. The above two approximations have been proved to be exact for some special cases; for example, the cases of the $M^{X} / G / 1 / N+1$ queue and the $M^{X} / M / c / N+c$ queue, and to be very accurate for many other cases. Based mainly on the numerical evidence, Miyazawa and Tijms [5] made the following conjecture.

Conjecture For both the $G I^{X} / G / 1 / N+1$ queue and the $G I^{X} / M / c / N+c$ queue it holds that

$$
P_{\text {appr }}(\text { cust }) \leq P_{\text {loss }} \leq P_{\text {appr }}(\text { time })
$$

if the interarrival time is NBUE (New Better than Used in Expectation), and

$$
P_{\text {loss }} \leq P_{\text {appr }}(\text { cust })
$$

if the interarrival time is NWUE (New Worse than Used in Expectation).

In the next section, we provide some counterexamples to this conjecture.

\section{Counterexamples}

In this section, we provide some counterexamples to the conjecture. Specifically, we consider the $G I / E_{r} / 1 / N+1$ queue. We are able to find counterexamples to the inequality $P_{\text {appr }}$ (cust) $\leq P_{\text {loss }}$ when the interarrival time is either Erlangian NBUE or generalized

Erlangian NBUE, and to the inequality $P_{\text {loss }} \leq P_{\text {appr }}$ (cust) when the interarrival time is generalized Erlangian NWUE. All examples are numerical as one might expect. 


\subsection{The $G I / E_{r} / 1$ queue}

This model has been analyzed by Adan and Zhao [1] based on the embedded Markov chain of the number of the uncompleted service stages just prior to an arrival. Let $\left\{\pi_{i}\right\}$ be the equilibrium distribution of this number. Then,

$$
\pi_{i}=\sum_{k=1}^{r} c_{k}\left(1-\sigma_{k}\right) \sigma_{k}^{i}, \quad i \geq 0
$$

where $\sigma_{k}$ are the roots of the characteristic equation of the model and $c_{k}$ can be explicitly expressed in terms of the roots (see equation (4) in [1]).

\subsection{The relationship between the $G I / E_{r} / 1$ queue and the $G I^{r} / M / 1$ queue}

The relationship between the $G I / E_{r} / 1$ queue and the $G I^{r} / M / 1$ queue has been discussed in the literature (for example, 6.3 of Chaudhry and Templeton [2]). Let $q_{i}^{-}, q_{i}$ and $q_{i}^{+}$be the equilibrium probabilities of $i$ customers in the system for the $G I / E_{r} / 1$ queue just prior to an arrival, at an arbitrary time epoch and just after a departure, respectively. Let $p_{i}^{-}$, $p_{i}$ and $p_{i}^{+}$be defined similarly for the $G I^{r} / M / 1$ queue. We then have

$$
\begin{gathered}
q_{i}^{-}= \begin{cases}p_{0}^{-}, & i=0, \\
\sum_{m=0}^{r-1} p_{i r-m}^{-}, & i>0\end{cases} \\
q_{i}^{+}=q_{i}^{-}, \quad i \geq 0
\end{gathered}
$$

and

$$
q_{i}= \begin{cases}p_{0}, & i=0, \\ \sum_{m=0}^{r-1} p_{i r-m}, & i>0,\end{cases}
$$

where

$$
p_{i}= \begin{cases}1-\rho, & i=0 \\ \rho p_{i-1}^{+}, & i>0\end{cases}
$$

with

$$
p_{i}^{+}= \begin{cases}\frac{1}{r} \sum_{m=0}^{i} p_{m}^{-}, & i<r \\ \frac{1}{r} \sum_{m=0}^{r-1} p_{i-m}^{-}, & i \geq r\end{cases}
$$

We also notice that

$$
\pi_{i}=p_{i}^{-}, \quad i \geq 0
$$




\subsection{Computation of $P$ appr (time) and $P$ appr (cust)}

The approximations $P$ appr (time) and $P$ appr (cust) are computed according to (1) and (2). It follows from the discussion in the previous subsection that in (1) and (2), $q_{i}^{-}$is computed according to (6) and (11), and $q_{i}$ is computed according to (8), (9), (10) and (11). Finally, $\pi_{i}$ are computed according to (5) using the method given in Adan and Zhao [1].

\subsection{Computation of the loss probability $\boldsymbol{P}_{\text {loss }}$}

Consider the embedded Markov chain of the number of the uncompleted service stages just prior to an arrival for the finite-buffer queue $G I / E_{r} / 1 / N+1$. Let $\left\{\pi_{i}^{(N)}\right\}$ be the equilibrium distribution of this number. The loss probability $P_{\text {loss }}$ is then given by

$$
P_{\text {loss }}=\sum_{i=N r+1}^{(N+1) r} \pi_{i}^{(N)} .
$$

$\pi_{i}^{(N)}$ are obtained by solving the stationary equations of the embedded Markov chain. A number of methods could be used for this purpose. The GTH method (see Grassmann, Taksar and Heyman [4]) is used here. Before using the GTH method, we need to compute all the transition probabilities. Since we only consider the case where the interarrival time is either Erlangian or generalized Erlangian, the transition probabilities can be explicitly expressed in terms of integrals of positive integrands. These integrals are approximated using the Gauss-Legendre method.

\subsection{Correctness of computations}

Since the counterexamples are given numerically, the correctness of all computations is crucial. Remember that we only consider the case where the interarrival time is either Erlangian or generalized Erlangian. The correctness of the computations rests on the following facts.

a) The accuracy of the computed transition probabilities relies on the approximation of the integrals. Since all the integrands are positive, the Gauss-Legendre method computes the integral up to a desired precision. A function written in $\mathrm{C}$ language implementing this method can be found in Numerical Recipes in C [8]. 
b) The GTH method is highly numerically stable. Also, in all of the examples used in the paper, $N$ and $r$ are relatively small, which means that the size of the transition matrix for $G I / E_{r} / 1 / N+1$ is relatively small.

c) The accuracy of the computed $\pi_{i}$ depends on the roots $\sigma_{i}$ of the characteristic equation of the model. This equation is rather simple when the interarrival time is either Erlangian or generalized Erlangian. In this case, $\sigma_{i}$ can be accurately computed without difficulty as discussed in Adan and Zhao [1].

\subsection{Examples}

Consider the generalized Erlangian interarrival time $X$ with probability density function

$$
f(t)=p \eta_{1}^{k_{1}} \frac{t^{k_{1}-1}}{\left(k_{1}-1\right) !} e^{-\eta_{1} t}+(1-p) \eta_{2}^{k_{2}} \frac{t^{k_{2}-1}}{\left(k_{2}-1\right) !} e^{-\eta_{2} t}, \quad t \geq 0
$$

where $p, \eta_{1}$ and $\eta_{2}$ are real numbers satisfying $0 \leq p \leq 1, \eta_{1}>0$ and $\eta_{2}>0$, and $k_{1}$ and $k_{2}$ are positive integers. The mean interarrival time is

$$
E(X)=p \frac{k_{1}}{\eta_{1}}+(1-p) \frac{k_{2}}{\eta_{2}} .
$$

Let

$$
H(\eta, k)=\sum_{j=0}^{k-1} \frac{(\eta t)^{j}}{j !} e^{-\eta t}
$$

then, after a long, but elementary, process of evaluations we find

$$
\begin{gathered}
{\left[p H\left(\eta_{1}, k_{1}\right)+(1-p) H\left(\eta_{2}, k_{2}\right)\right][E(X-t \mid X>t)-E(X)]=} \\
p(1-p)\left(\frac{k_{2}}{\eta_{2}}-\frac{k_{1}}{\eta_{1}}\right)\left[H\left(\eta_{2}, k_{2}\right)-H\left(\eta_{1}, k_{1}\right)\right]-(1-p) e^{-\eta_{2} t} \sum_{j=0}^{k_{2}-2} \eta_{2}^{j} \frac{t^{j+1}}{j !}-p e^{-\eta_{1} t} \sum_{j=0}^{k_{1}-2} \eta_{1}^{j} \frac{t^{j+1}}{j !},
\end{gathered}
$$

where on the right hand side, the third term equals zero if $k_{1}=1$ and the second term equals zero if $k_{2}=1$. The following result is an immediate consequence of the above equation.

Lemma 1 i) $X$ is NBUE when $k_{1} \eta_{2}=k_{2} \eta_{1}$; and ii) $X$ is $N W U E$ when $k_{1}=\eta_{1}=1, \eta_{2} \leq 1$ and $k_{2} \leq\left(1-p \eta_{2}\right) /(1-p)$.

Proof: The result in i) is obvious. To prove ii), rewrite the right hand side of (14) as

$$
p(1-p)\left(\frac{k_{2}}{\eta_{2}}-1\right)\left(e^{-\eta_{2} t}-e^{-t}\right)+\frac{1}{\eta_{2}}(1-p) \sum_{j=1}^{k_{2}-1} \frac{\left(\eta_{2} t\right)^{j}}{(j-1) !} e^{-\eta_{2} t}\left[\frac{p}{j}\left(k_{2}-\eta_{2}\right)-1\right] .
$$


It follows from $\eta_{2} \leq 1$ that

$$
p(1-p)\left(\frac{k_{2}}{\eta_{2}}-1\right)\left(e^{-\eta_{2} t}-e^{-t}\right) \geq 0
$$

It follows from $k_{2} \leq\left(1-p \eta_{2}\right) /(1-p)$ that

$$
p\left(k_{2}-\eta_{2}\right) \geq k_{2}-1 \geq j, \quad j=0,1, \ldots, k_{2}-1,
$$

or

$$
\frac{p}{j}\left(k_{2}-\eta_{2}\right)-1 \geq 0, \quad j=0,1, \ldots, k_{2}-1 .
$$

Therefore $X$ is NWUE.

Example 1 Consider the $G I / E_{r} / 1 / N+1$ queue where the interarrival time is a generalized Erlangian random variable whose density function is given by (13). Let $k_{1}=\eta_{1}=1$ and $k_{2}=\eta_{2}=5$. It follows from the lemma that the interarrival time is NBUE. Further, assume that $p=0.9, r=5, \rho=0.9$ and $N=5$. The loss probability and the approximation Pappr (cust) are computed as $P_{\text {loss }}=0.053067$ and $P$ appr $($ cust $)=0.053198$. It is a contradiction to the inequality $P_{\text {appr }}($ cust $) \leq P_{\text {loss }}$ conjectured.

Remark 1 a) When parameter values are changed, other counterexamples can also be found.

b) Counterexamples are found for a simpler interarrival time, one of which is given below.

Example 2 Consider the $G I / E_{r} / 1 / N+1$ queue where the interarrival time $X$ is a mixed Erlangian random variable whose density function is also given by (13) with $\eta_{1}=\eta_{2}$. Let $\eta_{1}=1, k_{1}=1, k_{2}=7$ and $p=0.9$. For this case, the interarrival time is NBUE. The idea is as follows. First, we show that there exist two real numbers $t^{\prime}$ and $t^{\prime \prime}$ with $0<t^{\prime}<t^{\prime \prime}$ such that $E(X) \geq E(X-t \mid X>t)$ for all $t$ satisfying $0<t<t^{\prime}$ or $t>t^{\prime \prime}$. We then divide the interval $\left[t^{\prime}, t^{\prime \prime}\right]$ into a number of subintervals with an equal length. When the length of subintervals is small enough, we can show that for any subinterval, $E(X) \geq$ $E(X-t \mid X>t)$ whenever $t$ is in that interval. Therefore, $X$ is NBUE. For this case, $P_{\text {appr }}($ cust $)=0.041329>P_{\text {loss }}=0.041282$. 
Remark 2 More examples can be found when we change values of the parameter. In this paper, we chose examples where probability values are relatively large. For these cases, contradictions to the conjecture cannot be caused by computational errors.

Example 3 In this example, we still consider the $G I / E_{r} / 1 / N+1$ queue where the interarrival time is a generalized Erlangian random variable whose density function is given by (13). Let $k_{1}=\eta_{1}=1, \eta_{2}=0.14, p=0.7$ and $k_{2}=3$. It follows from the lemma that the interarrival time is NWUE. Further, assume that $r=5, \rho=0.9$ and $N=5$. The loss probability and the approximation $P_{\text {appr }}($ cust $)$ are computed as $P_{\text {loss }}=0.180592$ and $P_{\text {appr }}($ cust $)=0.174218$. It is a contradiction to the inequality $P_{\text {appr }}($ cust $) \geq P_{\text {loss }}$ conjectured.

As concluding remarks, we would like to indicate that

a) We believe that a counterexample to the inequality $P_{\text {loss }} \leq P_{\text {appr }}$ (time) can be found. For the case where the interarrival time is generalized Erlangian, numerical results suggest that we may find such a counterexample if the traffic intensity $\rho$ is light and $N$ is not too small. However, if the traffic intensity is light and $N$ is not too small, then the loss probability is usually very small. We cannot be certain in this case if a contradiction to the conjecture is caused by computational errors. For example, when $k_{1}=\eta_{1}=1, k_{2}=\eta_{2}=8, p=0.4, r=5, N=10$ and $\rho=0.2$, the loss probability $P_{\text {loss }}$ and the approximation $P_{\text {appr }}($ time $)$ are computed as $P_{\text {loss }}=1.04718 \times 10^{-16}$ and $P$ appr $($ time $)=8.88178 \times 10^{-17}$. Computational errors in computing the roots $\sigma_{i}$ may lead these numbers to be incorrect.

b) We still do not have a complete answer to the conjecture even though we might be able to find a counterexample mentioned in a) since the conjecture may still hold for the $G I^{X} / M / c / N+c$ queue.

Acknowledgements: The author acknowledges that this work was supported by a research grant from the Natural Sciences and Engineering Research Council of Canada (NSERC) and also thanks I. Dell and R. El-Deeb for their help in numerically searching for counterexamples. The author especially acknowledges that an error in (14) in the previous version of the paper was pointed out by the referee, that avoided the use of an incorrect counterexample. 


\section{References}

[1] Adan, I., \& Zhao, Y. (1996). Analyzing GI/Er/1 queues. Operations Research Letters 19: $183-190$.

[2] Chaudhry, M.L., \& Templeton, J.G.C. (1983). A first course in bulk queues. New York: John Wiley \& Sons.

[3] Gouweleeuw, Frank N. (1996). A general approach to computing loss probabilities in finite-buffer queues. (Ph.D. thesis) Amsterdam: Thesis Publishers.

[4] Grassmann, W.K., Taksar, M.I., \& Heyman, D.P. (1985). Regenerative analysis and steady state distributions for Markov chains. Operations Research 33: 1107-1116.

[5] Miyazawa, M., \& Tijms, H.C. (1993). Comparison of two approximations for the loss probability in finite-buffer queues. Probability in the Engineering and Informational Sciences 7: 19-27.

[6] Sakasegawa, H., Miyazawa, M., \& Yamazaki, G. (1993). Evaluating the overflow probability using the infinite queue. Management Science 39: 1238-1245.

[7] Tijms, H.C. (1992). Heuristics for finite-buffer queues. Probability in the Engineering and Informational Sciences 6: 227-285.

[8] Vetterling, W.T., Teukolsky, S.A., Press, W.H., \& Flannery, B.P. (1992). Numerical recipes in $C$. Cambridge: Cambridge University Press. 